HEPSY 99-2

Feb. 1999

\title{
The CLEO-III Ring Imaging Cherenkov Detector
}

\author{
R.J. Mountain, M. Artuso, R. Ayad, F. Azfar, A. Efimov, S. Kopp, \\ G. Majumder, S. Schuh, T. Skwarnicki, S. Stone, G. Viehhauser \\ Syracuse University, Syracuse NY 13244-1130, USA \\ S. Anderson, A. Smith, Y. Kubota \\ University of Minnesota, Minneapolis MN 55455, USA \\ E. Lipeles \\ California Institute of Technology, Pasadena CA 91125, USA \\ T. Coan, J. Staeck, V. Fadeyev, I. Volobouev, J. Ye \\ Southern Methodist University, Dallas TX 75275, USA
}

\begin{abstract}
The CLEO-III Detector upgrade for charged particle identification is discussed. The RICH design uses solid LiF crystal radiators coupled with multi-wire chamber photon detectors, using TEA as the photosensor, and low-noise Viking readout electronics. Results from our beam test at Fermilab are presented.
\end{abstract}

Invited talk by R. Mountain at "The 3rd International Workshop on Ring Imaging Cherenkov Detectors," a research workshop of the Israel Science Foundation, Ein-Gedi, Dead-Sea, Israel, Nov. 15-20, 1998 


\section{INTRODUCTION}

The CLEO detector is undergoing a major upgrade (CLEO-III) in conjunction with a luminosity upgrade of the CESR electron-positron collider (CESR Phase-III) [1,2]. This upgrade will increase the luminosity of the machine by more than a factor of 10 , to $£=2 \times 10^{33} \mathrm{~cm}^{-2} \mathrm{sec}^{-1}$, or $\sim 20 \mathrm{fb}^{-1} / \mathrm{yr}$, allowing unprecedented sensitivity to study $\mathrm{CP}$ violation in charged $B$ decays as well as the phenomenology of rare $B$ decay modes (with $\mathrm{BR} \approx 10^{-6}$ ). Charged hadron identification is crucial in distinguishing these decay modes. Typically, one wants highly efficient $\pi / K$ separation with mis-identification probabilities

$\leq 10^{-2}$ over the full momentum range of secondaries from $B$-hadrons produced at the $\Upsilon(4 S)$ resonance. Achieving this capability in modern particle detection has heretofore been elusive.

We at CLEO believe that the best way to accomplish this task is to construct a Ring Imaging Cherenkov (RICH) Detector.

\section{GENERAL PRINCIPLES OF A PROXIMITY-FOCUSED RICH}

The CLEO-III RICH detector consists of three components: radiator, expansion volume, and photon detector. No focusing is used; this is called "proximity-focusing" [3]. When an incident charged particle with sufficient momentum $(\beta>1 / n)$ passes through a radiator medium, it emits photons at an angle $\Theta$ via the Cherenkov effect; some photons are internally reflected due to the large refractive index $n$ of the radiator, and some escape. These latter photons propagate in a transparent expansion volume, sufficiently large to allow the Cherenkov cone to expand in size (as much as other spatial constraints allow). The photons are imaged by a two-dimensional pad detector, a photosensitive multi-wire chamber which records their spatial position. The resulting images are portions of conic sections, distorted by refraction and truncated by internal reflection at the boundaries of media with different optical densities. Thus, knowing the track parameters of the charged particle and the refractive index of the radiator, one can reconstruct the Cherenkov angle $\Theta=\cos ^{-1}(1 / n \beta)$ and extract the particle mass.

This elegant and compact approach was pioneered by the Fast-RICH Group $[4-6]$.

In order to achieve efficient particle identification with low fake rates, we set as a design goal a system capable of $\pi / K$ separation with $4 \sigma$ significance $\left(N_{\sigma}=\Delta \Theta / \sigma_{\Theta}\right)$ at $2.65 \mathrm{GeV} / c$, the mean maximum momentum for twobody $B$-decays at a symmetric $e^{+} e^{-}$collider. At this momentum, the $\pi-K$ 
Cherenkov angle difference $\Delta \Theta=14.4 \mathrm{mrad}$, which along with $1.8 \sigma d E / d x$ identification from the central Drift Chamber, requires a Cherenkov angle res-

olution $\sigma_{\Theta}=4.0 \mathrm{mrad}$ per track. Using the relation $\sigma_{\Theta}=\sigma_{\Theta \mathrm{pe}} / \sqrt{N_{\text {pe }}}$, we can establish round-number benchmarks for our design: a resolution of $14 \mathrm{mrad}$ per photoelectron [7], and a photoelectron yield of 12 pe per track.

\section{BASIC DETECTOR DESIGN}

The overall RICH design is cylindrical, with compact photon detector modules at the outer radius and radiator crystals at the inner radius, forming thirty $12^{\circ}$ sectors in azimuth. A schematic of the RICH is given in Ref. [1]. The RICH resides between the central Drift Chamber and the CsI Calorimeter. The ensuing space budget constrains the detector to fit in 80-100 cm in radius, and $2.5 \mathrm{~m}$ in length ( $82 \%$ of the solid angle). The mass budget restricts the thickness to $12 \% X_{\mathrm{o}}$ to avoid significantly degrading the performance of the Calorimeter.

However, the driving constraint of the design in many respects is the choice of Triethylamine (TEA) as a photosensor, which is both chemically aggressive and manifests a quantum efficiency in the VUV regime $(135-165 \mathrm{~nm})$. This greatly restricts the available materials: optical materials must be transparent in the VUV, and construction materials need to be chemically resistant to TEA and low outgassing.

Each detector component and related design issues are discussed in turn.

\section{CRYSTAL RADIATORS}

The baseline design for the radiator is an array of individual planar LiF crystals $\square$, each $\sim 170 \times 170 \mathrm{~mm}^{2}$ and $10 \mathrm{~mm}$ thick, mounted on an inner carbon fiber cylinder. Due to the high refractive index of $\operatorname{LiF}(n=1.50$ at $150 \mathrm{~nm})$, a large number of Cherenkov photons are internally reflected, resulting in only a partial ring being imaged (about $1 / 3$ of the initial cone). This is especially severe for incident track angles under $15^{\circ}$, where the radiator would have to be tilted on the inner cylinder for the incident track to exceed this angle.

In order to improve this situation, a novel radiator geometry has been implemented [7], cf. Figure 1. This "sawtooth" radiator, with its inner surface cut

1 The $\mathrm{LiF}$ and $\mathrm{CaF}_{2}$ crystals are grown and polished by OPTOVAC, North Brookfield, MA. 


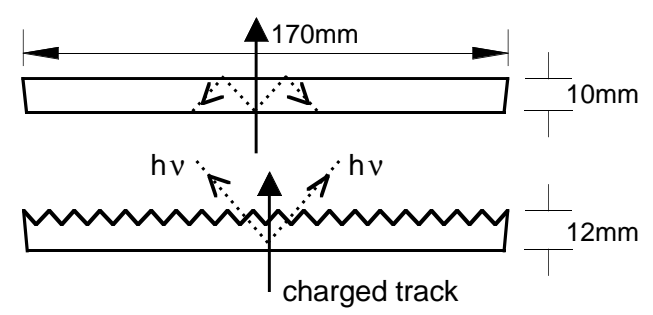

Fig. 1. Azimuthal view of planar and sawtooth radiators with a normal incidence track.

in profile to resemble the teeth of a saw, allows photons to cross the surface at near normal incidence. This reduces the photon loss by internal reflection and, as a consequence of the refraction angle, also the dominant chromatic error contribution to the resolution. Detailed Monte Carlo simulations [7] indicate that all performance parameters are better with a sawtooth radiator, and especially so at small values of incident track angle. Hence the central region of the detector will use 120 sawtooth radiators (47\% of the solid angle coverage), and the outer regions will use 300 planar radiators.

\section{EXPANSION VOLUME}

The expansion volume is essentially empty space, $157 \mathrm{~mm}$ in radial distance, filled with pure $\mathrm{N}_{2}$ gas. There are no support structures in the interior of the detector, which would be obstructions to photon propagation. However structural rigidity must be maintained with low mass, and so our mechanical design calls for aluminum end-flanges glued to the inner cylinder, and a detector module support frame, with reinforcing rib and box structures added to the modules.

Importantly, UV photons will be lost if the expansion volume is not well-sealed from $\mathrm{O}_{2}$ and $\mathrm{H}_{2} \mathrm{O}$ contamination, and from any possible leakage from the photon detector volume. Redundant gas seals are used in our design, and the $\mathrm{N}_{2}$ will be exchanged at a flow rate sufficient to maintain high transparency.

\section{PHOTON DETECTORS}

The photon detector is a compact, photosensitive asymmetric multi-wire chamber, shown in Figure 2, filled with $\mathrm{CH}_{4}$ carrier gas bubbled through liquid TEA at $15^{\circ} \mathrm{C}$ (5.5\% vapor concentration). TEA has a peak QE of $33 \%$ at $150 \mathrm{~nm}$ and a spectral bandwidth of $135-165 \mathrm{~nm}$ [6]. The detection sequence is: a photon passes through a thin UV-transparent $\mathrm{CaF}_{2}$ window and is converted to 


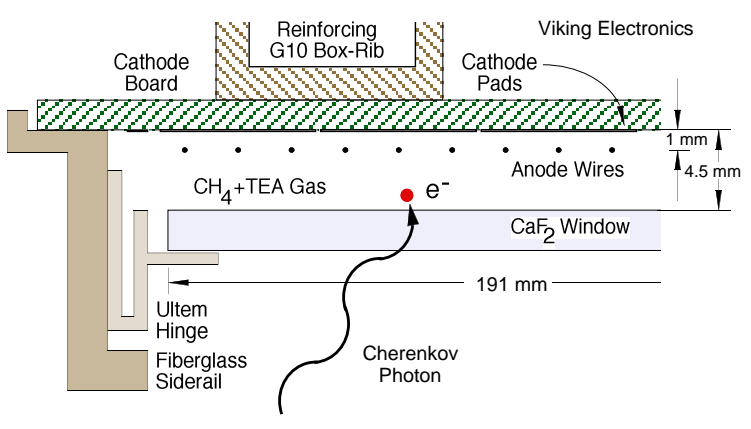

Fig. 2. Schematic cross-section of a single photon detector module. A Cherenkov photon is shown propagating from the radiator.

a single electron by ionizing a TEA molecule. The single photoelectron drifts towards, then avalanches near, the $20 \mu \mathrm{m} \varnothing \mathrm{Au}-\mathrm{W}$ anode-wires, and induces a charge signal on the array of $8.0 \times 7.5 \mathrm{~mm}^{2}$ cathode-pads, providing a spatial point for the photoelectron. The design maximizes the photon conversion efficiency by having the wire-window gap be many photoabsorption lengths ( $\epsilon=99.9 \%$, with $\ell_{\mathrm{abs}}=0.5 \mathrm{~mm}$ at $\left.150 \mathrm{~nm}\right)$, and maximizes the anode-cathode charge coupling $(C \approx 78 \%)$ by having the wire-pad gap be as small as practical $(1 \mathrm{~mm})$. Both may be optimized at once for a fixed thickness by making the chamber asymmetric.

The $\mathrm{CaF}_{2}$ window crystals must be deposited with metallized traces in order to act as field electrodes. Moreover, the crystals are large $\left(191 \times 308 \mathrm{~mm}^{2}\right)$ and quite thin $(2 \mathrm{~mm})$ in order to minimize photon absorption and radiation length, and must be mounted with no mechanical stress to avoid inducing fractures. Our design utilizes Ultem hinges, to which the $\mathrm{CaF}_{2}$ windows are glued, to relieve stresses. All construction materials in the chamber volume must be low outgassing and TEA-compatible. Extensive tests of TEA effects on various materials and adhesives of interest have been made.

\section{READOUT ELECTRONICS}

The choice of readout electronics is governed by the Furry (exponential) charge distribution of a single photoelectron avalanche, cf. Figure 3, and also by the time allowed for the readout. The most likely charge is zero, but the tail is long, and so it is necessary to have analog front-end electronics with low noise and large dynamic range in order to maximize the photoelectron detection efficiency. The charge information is necessary in accurately determining the centroid of the photoelectron, as well as in disentangling the overlap of two nearby charge distributions. The latter requires high segmentation. Given the modest pad size over the large detector area, there are 230,400 total electronics 


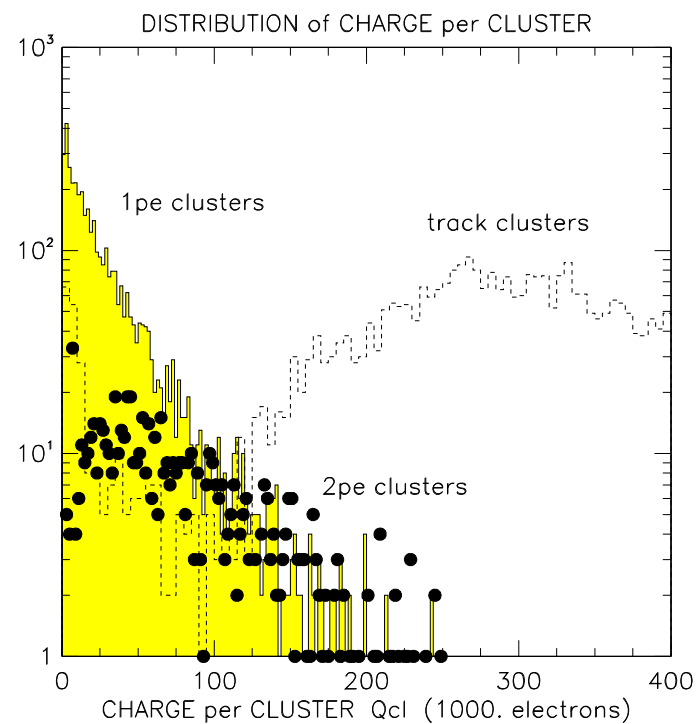

Fig. 3. Distribution of charge per reconstructed cluster: for single-photoelectron clusters, two-photoelectron clusters, and charged-track clusters; on plateau, in $\mathrm{CH}_{4}+\mathrm{TEA}\left(15^{\circ} \mathrm{C}\right)$. Data from cosmic-ray tests.

channels. Occupancy is low $(<1 \%)$ and so sparsification is required.

The front-end signal processor is the Viking [8] VA_RICH chip, a new customdesigned 64-channel VLSI chip ${ }^{2}$ incorporating these requirements, with measured rms noise $\mathrm{ENC}=130 e^{-}+9 C_{\mathrm{det}} e^{-} / \mathrm{pF}\left(\approx 150 e^{-}\right.$for modest $\left.C_{\mathrm{det}} \approx 2 \mathrm{pF}\right)$, a shaping time of $1-3 \mu \mathrm{sec}$, and good linearity up to $\pm 4.5 \times 10^{5} e^{-}$input. The chip has input protection, a preamplifier/shaper, sample $\&$ hold circuitry, and a differential current output multiplexer. The analog signal travels over a $6 \mathrm{~m}$ long cable from the detector to a VME databoard with receiver, 12-bit ADC and sparsifier.

\section{BEAM-TEST SETUP}

In order to test our understanding of the design and behavior of this detector, a comprehensive beam test was performed. The first two completed photon detectors of the CLEO-III RICH were mounted on an aluminum box simulating the expansion volume, and equipped with one planar and two sawtooth LiF radiators (cf. Figure 4).

The beam test [9] was performed in a muon halo beam in the Meson East area of Fermilab, downstream of Experiment E866. The setup consisted of the

$\overline{2}$ This Viking chip was designed and manufactured by IDE AS, Oslo, Norway. 


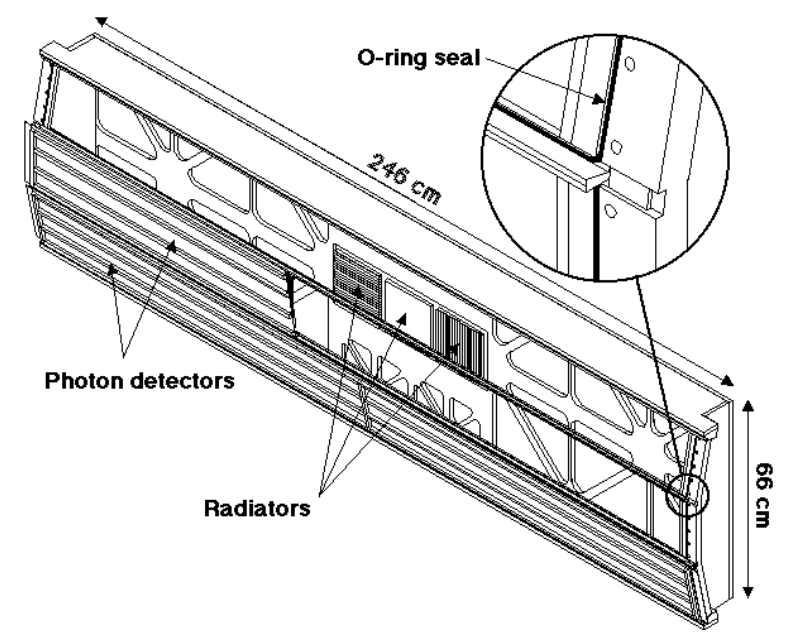

Fig. 4. RICH beam-test box. Half of the middle chamber is cut away to show the radiators. The outer radiators were sawtooth radiators with the teeth arranged perpendicularly to each other.

RICH itself, its supporting gas and HV systems, trigger scintillators, and a charged-particle tracking system $(2$ MWPCs with $0.7 \mathrm{~mm}$ spatial resolution per station and combined $\sim 1$ mrad track angle resolution). Data was taken with the RICH box rotated at various polar and azimuthal angles to simulate the different incident track angles expected in CLEO-III.

Photon detector operation was stable during the three week beam test, running at a nominal gain of $4 \times 10^{4}$. The readout for the beam test consisted of 240 VA_RICH chips and 8 VME databoards (for 15360 channels). After commonmode subtraction the remaining incoherent rms noise observed was $400 e^{-}$, providing an average signal-to-noise ratio for photoelectrons of 100:1.

\section{BEAM-TEST RESULTS}

Photoelectrons (pe) are reconstructed by determining topological clusters of pads with pulse height above a $5 \sigma$ pedestal cut $\left(\sigma=400 e^{-}\right)$. The overlap of multiple photoelectrons in a given cluster is disentangled by using the pulse height profile. The unbiased centroid is then found as the location of the photoelectron. (At operating voltage, pad multiplicities are 2.2 pads per cluster, and 1.1 pe per cluster.) From each photoelectron position, the original photon trajectory is optically traced back through all media to the center of the radiator, and the Cherenkov angle is reconstructed. Charged track clusters are distinguished from photon clusters by total charge and by the number of pads in the cluster. 
Data has been taken at a variety of track angles; in the following discussion only the datasets for the plane radiator at $30^{\circ}$ track incidence and for the sawtooth radiator at $0^{\circ}$ are considered in detail.

Figure 5 shows a cumulative event display for all ring images in these two datasets. For the plane radiator one arc of the Cherenkov ring is visible, while for the sawtooth radiator two arcs in opposition are visible, with the lower one largely outside of the fiducial region of the detectors. Acceptance is lowered by this image truncation, and by mechanical transmission losses from construction elements in the detector. The acceptance for contained plane radiator images is the maximum realistic acceptance for a full RICH system, which is about $85 \%$; the acceptance for sawtooth images is approximately $50 \%$ in the two-sector beam test setup.

Results from the analysis of the $30^{\circ}$ plane radiator dataset are shown in Figure 6; only images confined to a single detector are used. The distribution of Cherenkov angle for single photoelectrons has an asymmetric tail and modest background; it is fit with a Crystal-Ball [3 lineshape plus polynomial background, yielding a single photoelectron Cherenkov angle resolution $\sigma_{\Theta p e}=(13.2 \pm 0.05 \pm 0.56)$ mrad with a background fraction of $9.2 \%$ under the image and a Monte Carlo estimate of $13.5 \mathrm{mrad}$. Errors quoted are first statistical then systematic, with the latter taken from two different fitting procedures, i.e., two methods of background estimation. This background is not electronic noise but rather it is principally due to out-of-time hadronic showers from an upstream beam dump; there will be no such background in the CLEO-III running conditions.

The Cherenkov angle per track is found as the arithmetic mean of all photoelectrons in an image. There is an image cut of $\pm 3 \sigma_{\Theta \text { pe }}$ and a systematic alignment correction applied. The resulting distribution of Cherenkov angle per track is fit to a Gaussian, and gives the angle resolution per track $\sigma_{\Theta \text { trk }}=(4.54 \pm 0.02 \pm 0.23) \mathrm{mrad}$, which compares favorably with the Monte Carlo estimate of $4.45 \mathrm{mrad}$. The systematic error is estimated from the variation ${ }^{4}$ between different datasets taken at the same track angle, which are repeatable to $5 \%$.

The photoelectron yield $N_{\text {pe }}=(12.9 \pm 0.07 \pm 0.36)$ pe per track is extracted from the area under the single photoelectron peak followed by background subtraction. Again systematic errors dominate and are given by different methods

3 The Crystal-Ball lineshape is a Gaussian with an exponential tail at higher angles. Resolution $(\sigma)$ is extracted from the full-width at half-maximum.

4 This variation has a number of root causes, each at the few percent level: the expansion volume transparency was monitored to be above $95 \%$, there are variations in transparency over each radiator face, etc. Hence the systematic error is estimated to be at the $5 \%$ level. 
of background estimation. (Here the beam-test Monte Carlo makes no prediction for $N_{\text {pe }}$ but rather uses the measurement as an input parameter.) This yield exceeds our benchmark of 12 pe/track.

Similar analysis for the $0^{\circ}$ sawtooth radiator dataset, cf. Figure 7 , gives a single photoelectron Cherenkov angle resolution $\sigma_{\Theta \text { pe }}=(11.7 \pm 0.03 \pm 0.42)$ mrad with a background fraction of $12.0 \%$ (compared with $11.1 \mathrm{mrad}$ from Monte Carlo), an angle resolution per track $\sigma_{\Theta \text { trk }}=(4.49 \pm 0.01 \pm 0.22) \mathrm{mrad}(4.28 \mathrm{mrad}$ from Monte Carlo), and a photoelectron yield $N_{\mathrm{pe}}=(10.4 \pm 0.04 \pm 1.0)$ pe/track, background subtracted. Adjusted for full $85 \%$ geometric acceptance, $N_{\text {pe }}$ becomes 18.8 pe/track.

Figure 8 provides a summary of beam-test results from all datasets at all incident angles. The measured Cherenkov angle resolution per track from the plane radiator data (denoted by squares) increases as a function of the incident track angle due to the increase in emission-point error. 0 The beam-test Monte Carlo simulation gives the light dashed curve in Figure 8, which represents the data well.

However the per track resolution, e.g. $4.54 \mathrm{mrad}$ at $30^{\circ}$, is larger than that naively calculated by statistics, i.e. $13.2 \mathrm{mrad} / \sqrt{12.9}=3.68 \mathrm{mrad}$. Monte Carlo studies indicate that the sources of the increased resolution are the MWPC tracking errors (the principal cause, $2.3 \mathrm{mrad}$ at $30^{\circ}$ ) and the beam background $\left(1.2 \mathrm{mrad}\right.$ at $\left.30^{\circ}\right)$. The tracking errors per se obviously cannot change with rotation of the RICH box, rather they effectively increase the emission-point error due to an incorrect track impact point on the radiator face, and hence become more prominent with track angle. In CLEO-III the background will be much reduced, and the tracking error contribution will be smaller yet still significant.

In order to estimate the ultimate performance of this RICH, an extrapolation was made based on the beam-test Monte Carlo. The background and tracking errors are associated only with our beam test, so both were removed from the simulation for this study. The resulting photoelectron yield was then corrected for the geometric acceptance of the beam-test setup and scaled to "full acceptance", defined as $85 \%$ of the solid angle covered by a cylindrical RICH. The result of this "full acceptance" extrapolation for the per track resolution for the plane radiator is shown as the light solid curve in Figure 8, which is flat in track angle and below our benchmark of $4 \mathrm{mrad}$ for CLEO-III.

The measured per track resolution from the sawtooth radiator data (denoted by circles in Figure 8) also increases with track angle, as expected [7], again

5 The Cherenkov angle resolution per track is dominated by chromatic and emission-point errors [3]. The chromatic error is larger at small track angles, but they become comparable at large track angles. 
due to the increase in emission-point error. However the value of the measured per track resolution is larger than expected. This has several sources: acceptance, MWPC tracking errors, beam background, and sawtooth profile effects. Geometric acceptance is the largest contribution; it is approximately $50 \%$ for all track angles. By naive statistical calculation this increases the per track resolution by 35\%. Monte Carlo studies show that tracking errors are the next largest contribution (e.g. $1.9 \mathrm{mrad}$ at $0^{\circ}$ ), and are exacerbated in this configuration because one of the arcs in the image is out of the detector fiducial. The beam background is approximately constant for all track angles (e.g. $1.3 \mathrm{mrad}$ at $0^{\circ}$ ). Sawtooth profile effects are defined as deviations of the real sawtooth radiator from an ideal sawtooth (e.g. rounding of the edges of teeth). However our simulation indicates that profile effects contributes little to the broadening of the resolution since mechanical imperfections are offset by a reduced transmission through the radiator in these same areas.

However even taking into account all these effects the beam-test Monte Carlo, which gives the heavy dashed curve in Figure 8, does not represent the data completely. It consistently underestimates the resolution, indicating that there are additional subtle systematic effects associated with the sawtooth radiator yet to be investigated.

The "full acceptance" extrapolation for the per track resolution for the sawtooth radiator, the heavy solid curve in Figure 8, is flatter in track angle and closer to our expectations in value.

A more sophisticated approach is made in Figure 9, which shows the Cherenkov resolution per track for the $0^{\circ}$ sawtooth dataset as a function of photoelectron yield. One may read off the per track resolution at the measured yield of 10.4 pe/track and extrapolate to the expected yield of 18.8 pe/track, giving the result as $3.5 \mathrm{mrad}$. Since this curve is derived from the data it automatically takes into account statistical and systematic effects. Hence we have met our benchmark of $4 \mathrm{mrad}$.

To summarize, for both plane and sawtooth radiators unfolding the effects of background and tracking error gives angle resolution per track very close to the expected values. The expected CLEO-III RICH performance will fall somewhere between the beam-test Monte Carlo curve and the "full acceptance" curve in Figure 8. Clearly this is sufficient to meet our needs for CLEO-III.

\section{CONCLUSIONS and OUTLOOK}

A beam test of the first two sectors of the CLEO-III RICH Detector has been successfully carried out. The results obtained fulfill CLEO-III requirements 
for $4 \sigma \pi / K$ separation, particularly a Cherenkov angle resolution of about 4 mrad.

The CLEO-III RICH Detector is in the final phase of construction. At present $85 \%$ of the photon detectors have been built (with $40 \%$ fully tested); all $\mathrm{CaF}_{2}$ windows, $78 \%$ of the LiF planar radiators, and $51 \%$ of the LiF sawtooth radiators have been delivered; and all readout chips have been acquired and tested. Completion and installation is expected in Summer 1999.

\section{ACKNOWLEDGMENTS}

We would like to thank Fermilab for providing us with the dedicated beam time for our test, the Computing Division for its excellent assistance, and our colleagues from E866 for their hospitality in the beamline.

\section{References}

[1] S.E. Kopp, Nucl. Instr. Meth. A384 (1996) 61.

[2] M. Artuso, "Progress Towards CLEO III", to be published in the Proceedings of the XXIX International Conference on High Energy Physics, hep-ex/9811031 (1998).

[3] T. Ypsilantis and J. Séguinot, Nucl. Instr. Meth. A343 (1994) 30.

[4] R. Arnold et al., Nucl. Instr. Meth. A314 (1992) 465.

[5] J.-L. Guyonnet et al., Nucl. Instr. Meth. A343 (1994) 178.

[6] J. Séguinot et al., Nucl. Instr. Meth. A350 (1994) 430.

[7] A. Efimov et al., Nucl. Instr. Meth. A365 (1995) 285.

[8] E. Nygard et al., Nucl. Instr. Meth. A301 (1991) 506.

[9] G. Viehhauser et al., Nucl. Instr. Meth. A419 (1998) 577. 

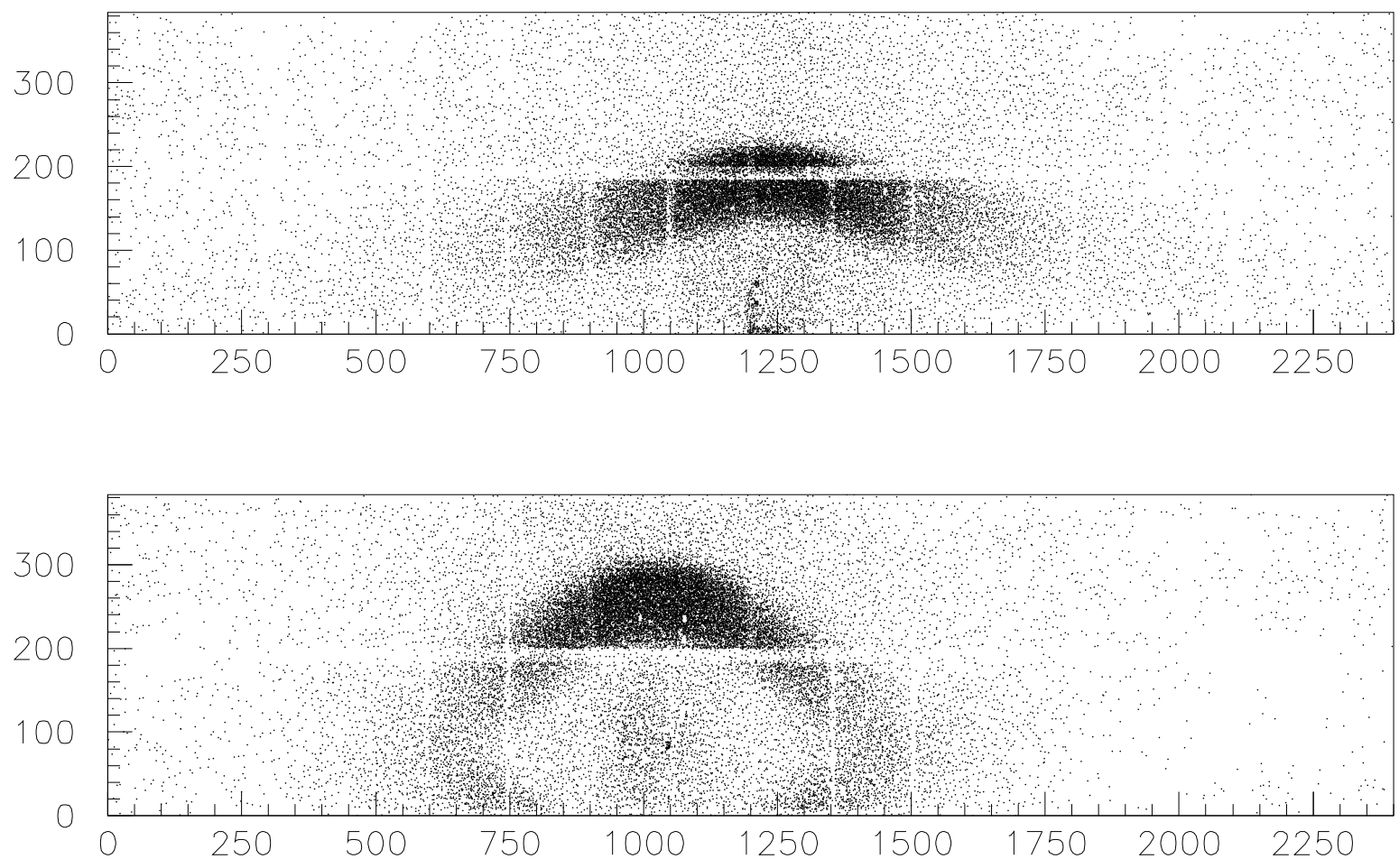

Fig. 5. Cumulation of 2489 Cherenkov images in (upper) $30^{\circ}$ plane radiator dataset, and 7434 images in (lower) $0^{\circ}$ sawtooth radiator dataset. Units are $\mathrm{mm}$. The bottom chamber contains the beam track, and is parallel to the radiator. Shadows of structural elements of the photon detectors can be seen. 

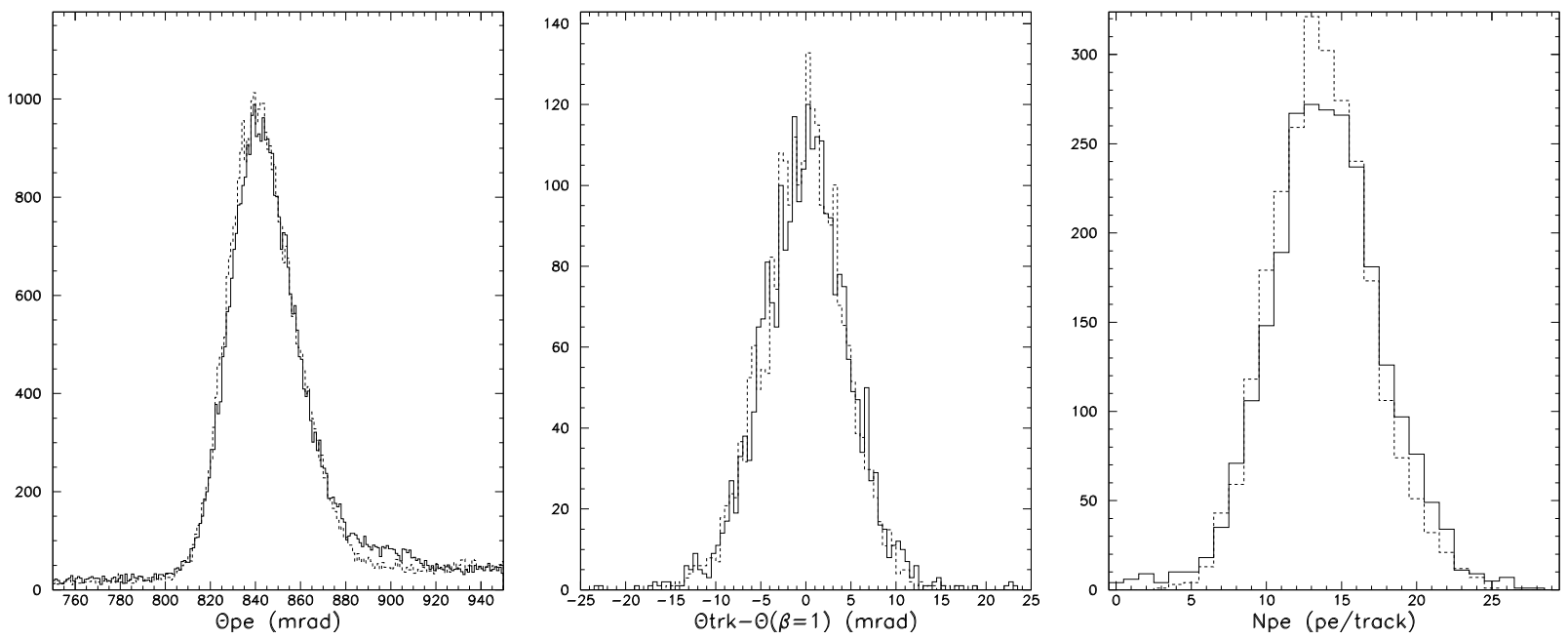

Fig. 6. Plane radiator results. (a) Single photoelectron Cherenkov angle distribution; (b) Distribution of Cherenkov angle per track shifted by angle of a high momentum muon $\left(\Theta_{\beta=1}\right)$; (c) Photoelectron yield (pe/track). Solid line is for data, dashed line is for Monte Carlo.
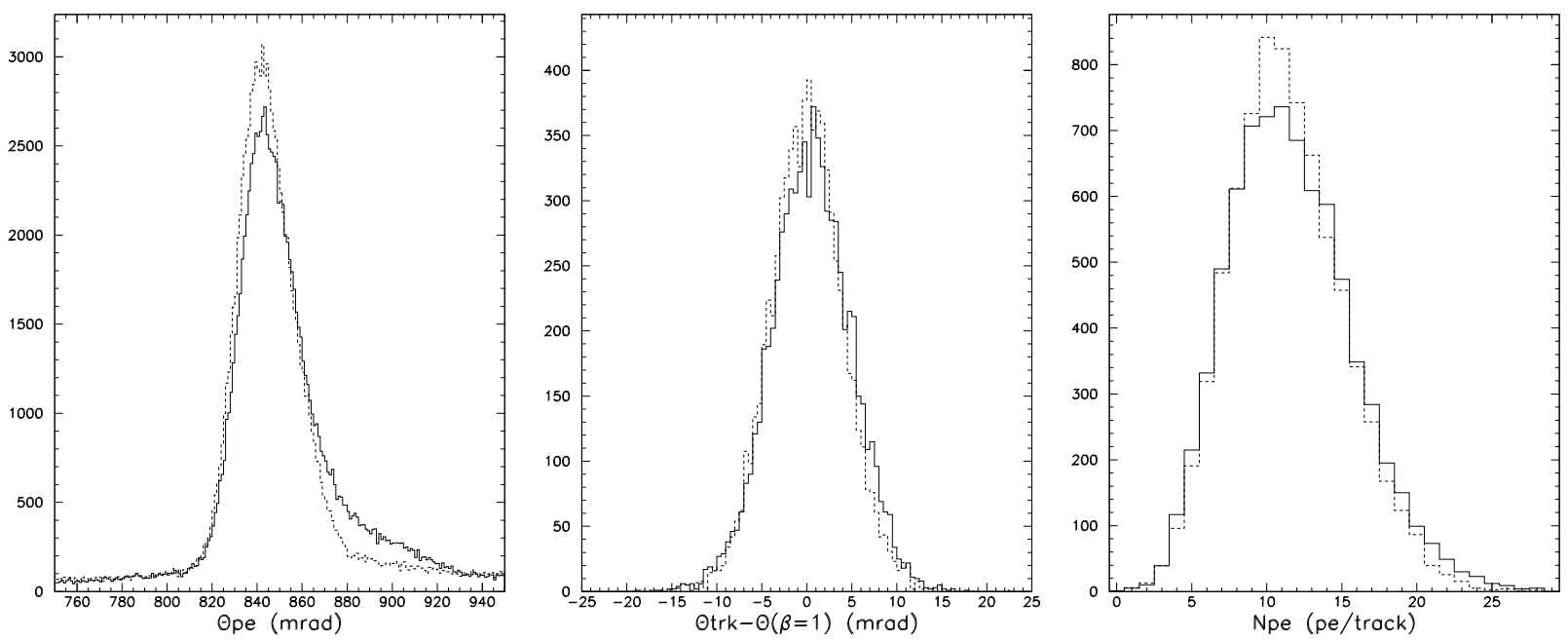

Fig. 7. Sawtooth radiator results. (a) Single photoelectron Cherenkov angle distribution; (b) Distribution of Cherenkov angle per track shifted by angle of a high momentum muon $\left(\Theta_{\beta=1}\right)$; (c) Photoelectron yield (pe/track). Solid line is for data, dashed line is for Monte Carlo. 


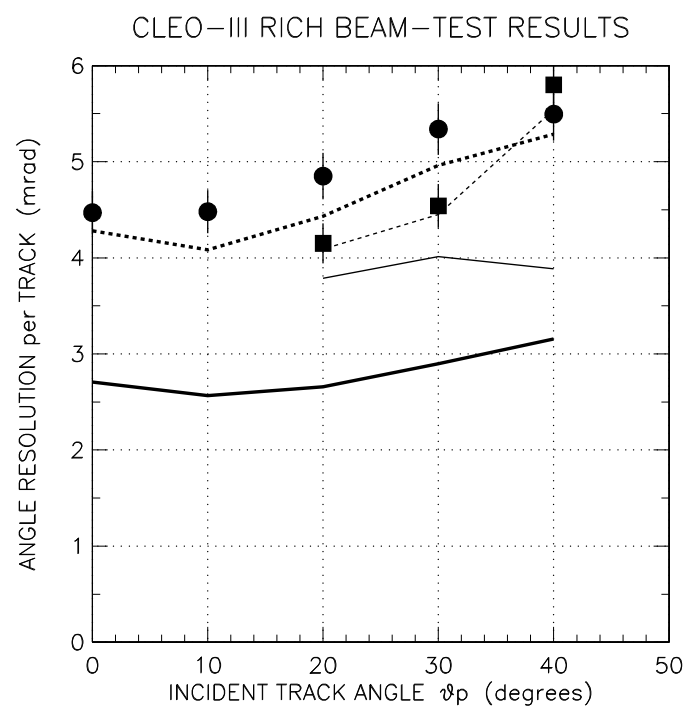

Fig. 8. Summary of CLEO-III RICH beam-test results. Squares indicate plane radiator results, and circles sawtooth radiator results. The filled symbols represent results from beam-test data, dashed curves results from beam-test Monte Carlo, and solid curves results from the "full acceptance" extrapolation.

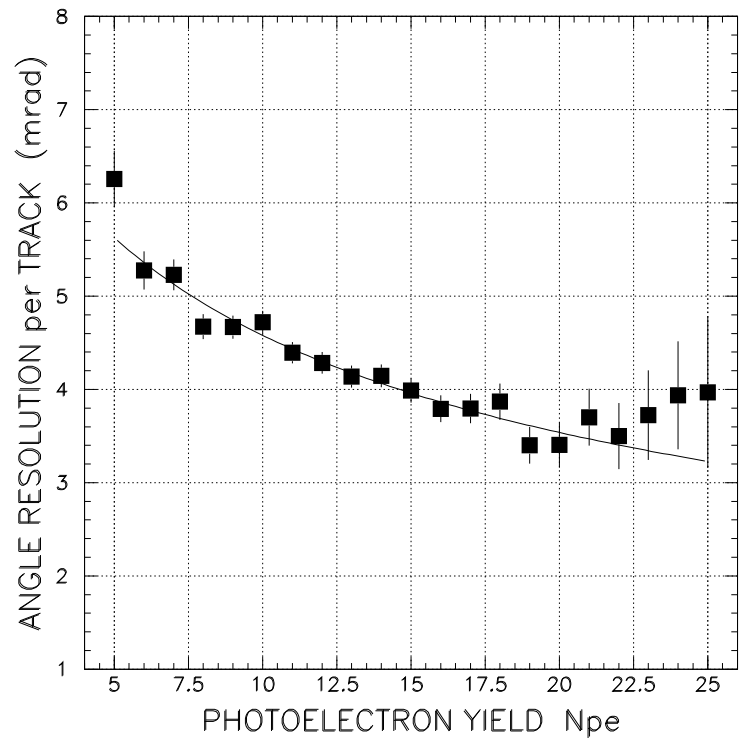

Fig. 9. The Cherenkov angle resolution per track as a function of photoelectron yield for sawtooth data at $0^{\circ}$ track incidence. The curve is a fit to $A+B / \sqrt{N_{\mathrm{pe}}+C}$. 